\title{
La surveillance de la rougeole au Canada : Tendances 2014
}

\author{
Sherrard $L^{1 *}$, Hiebert $J^{2}$, Squires $S^{1}$ \\ ${ }^{1}$ Centre de l'immunisation et des maladies respiratoires infectieuses, Agence de la santé publique du Canada, Ottawa \\ (Ontario) \\ ${ }^{2}$ Laboratoire national de microbiologie, Agence de la santé publique du Canada, Winnipeg (Manitoba) \\ *Correspondance : lindsey.sherrard@phac-aspc.gc.ca
}

\section{Résumé}

Contexte : Le statut d'élimination de la rougeole a été atteint au Canada en 1998. L'Agence de la santé publique du Canada compile des données probantes à l'intention de l'Organisation panaméricaine de la santé afin de confirmer que les critères pour la vérification de l'élimination de la rougeole continuent d'être satisfaits.

Objectif : Faire état de l'activité rougeoleuse au Canada en 2014 afin de valider le statut actuel d'élimination de la rougeole au Canada.

Méthodologie : Évaluation de la distribution des cas de rougeole en fonction des données démographiques, de l'état vaccinal et des hospitalisations; production d'un résumé sur les caractéristiques des éclosions; et analyses génotypiques et phylogéniques, à partir des données saisies par le Système canadien de surveillance de la rougeole et de la rubéole et le projet pilote sur la surveillance de la rougeole et de la rubéole durant 2014.

Résultats : Durant 2014, 418 cas de rougeole ont été déclarés dans cinq provinces/territoires, ce qui représente un taux d'incidence global de 11,8 pour 1000000 habitants. Le nombre de cas et les taux d'incidence étaient le plus élevés chez les enfants et les adolescents âgés de 5 à 14 ans, et la majorité des cas n'étaient pas immunisés. Cinq pour cent de l'ensemble des cas ont été hospitalisés; il s'agissait le plus souvent de patients dans les groupes d'âge plus jeunes ou plus vieux. Dix-huit éclosions ont été déclarées, la plus grande survenant dans une communauté religieuse ne pratiquant pas la vaccination en Colombie-Britannique. Les renseignements sur le génotype étaient accessibles pour $98 \%$ des cas de rougeole (18 éclosions sur 18, et 31 cas sporadiques sur 32). Le Canada continue de satisfaire, pleinement ou partiellement, aux quatre critères de l'Organisation panaméricaine de la santé pour la vérification de l'élimination de la rougeole.

Conclusion : Malgré une activité importante de la rougeole en 2014, le Canada continue de fournir de solides preuves que son statut d'élimination de la rougeole est maintenu.

\section{Introduction}

Le statut d'élimination de la rougeole a été obtenu au Canada en 1998, un an après le dernier cas endémique signalé en 1997. (1) Depuis ce jour, l'Agence de la santé publique du Canada (l'Agence) cumule toutes les preuves nécessaires pour assurer la vérification continue du statut d'élimination de la rougeole au Canada (1, 2 , 3). L'élimination de la rougeole est définie comme l'absence de transmission de la rougeole endémique dans une zone géographique définie ( $p$. ex. région) pendant $\geq 12$ mois au moyen d'un système de surveillance bien rodé (4). Les preuves proviennent de données de surveillance recueillies par des systèmes de surveillance accrue, et sont soutenues par diverses méthodes moléculaires. Le génotypage contribue à la fois au suivi de la santé publique et aux efforts continus d'élimination, et ce, grâce aux renseignements établissant des liens entre les cas, à l'identification des sources possibles d'importation, ainsi qu'à la démonstration de l'absence de tout génotype en circulation continue (c.-à-d. absence de transmission endémique) (5).

Même si on a réussi à interrompre la transmission du virus de la rougeole endémique dans la Région des Amériques en 2002 (6), la transmission endémique subsiste dans cinq autres régions de l'Organisation mondiale 
de la Santé (OMS), soit l'Afrique, la Méditerranée orientale, l'Europe, l'Asie du Sud-Est et le Pacifique occidental. Le Canada continue donc de courir le risque d'une importation du virus de la rougeole. En outre, malgré une couverture vaccinale estimée élevée au Canada (7), des éclosions de rougeole continuent de se produire (8), puisque la couverture vaccinale n'est pas homogène. Afin d'améliorer la documentation et la vérification de l'état d'élimination de la rougeole, l'Organisation panaméricaine de la santé (OPS) a établi quatre critères essentiels (voir l'encadré ci-dessous).

Quatre critères essentiels pour la vérification de l'élimination de la rougeole (9)

1. Vérifier l'interruption des cas endémiques de rougeole au cours d'une période d'au moins trois ans à compter du dernier cas endémique connu, en présence d'une surveillance de qualité supérieure.

2. Maintenir un système de surveillance de qualité supérieure suffisamment sensible pour déceler les cas importés et ceux liés à l'importation.

3. Vérifier l'absence de souches de virus endémiques de la rougeole en assurant une surveillance virologique.

4. Veiller à ce que l'immunisation au sein de la population soit adéquate.

\section{Méthodologie}

\section{Ensemble des données de surveillance}

Le Système canadien de surveillance de la rougeole et de la rubéole (SCSRR) est un système actif de surveillance accrue présent dans toutes les provinces et tous les territoires canadiens. Chaque semaine, les provinces et les territoires sont invités par l'Agence à soumettre un rapport sur le nombre de cas de rougeole confirmés durant ladite semaine de déclaration, ou un rapport de déclaration nulle. Trois régions (ColombieBritannique, Alberta et Terre-Neuve-et-Labrador) ont également pris part au projet pilote sur la surveillance de la rougeole et de la rubéole, une plateforme en ligne et en temps quasi réel pour la déclaration des enquêtes de cas suspects de rougeole. Tous les cas étaient évalués au moyen de la définition nationale de cas (10).

Ce rapport décrit les données améliorées sur les cas confirmés de rougeole accompagnés d'une éruption cutanée apparue au cours de l'année épidémiologique 2014 (semaines 1 à 53, du 29 décembre 2013 au 3 janvier 2015), tels que déclarés par les provinces et les territoires.

\section{Formulaire de déclaration de cas}

Les données du SCSRR étaient recueillies au moyen du formulaire national de déclaration de cas de rougeole, lequel colligeait les renseignements nécessaires pour appuyer les objectifs nationaux de surveillance de la rougeole dans les catégories suivantes : identificateurs de cas; contexte, exposition et renseignements cliniques; et renseignements de laboratoire. Le formulaire national de déclaration de cas est accessible en ligne (11).

\section{Génotypage}

Tous les génotypages du virus de la rougeole ont été effectués au Laboratoire national de microbiologie (LNM) de l'Agence. Des échantillons cliniques appropriés (respiratoires et/ou urinaires) recueillis auprès de cas suspects ou confirmés de rougeole ont été soumis au LNM par les laboratoires provinciaux. La région de génotypage normalisée de l'OMS, soit les 450 nucléotides qui codent l'extrémité carboxylique de la nucléoprotéine de la rougeole, la région $\mathrm{N}-450$ (12), a été amplifiée et séquencée à partir d'extraits d'acides nucléiques. Les séquences obtenues ont été alignées sur les séquences de référence des génotypes de l'OMS (13) et des arbres phylogénétiques de parcimonie maximale étaient générés au moyen du logiciel MEGA6 (14).

\section{Gestion des données et analyse statistique}

Les données du SCSRR ont été gérées à l'aide du logiciel Access de Microsoft de 2010. Les champs vides ont été codés comme des valeurs manquantes, tandis que les champs pour lesquels une valeur était requise mais non disponible dans la source de données, ont été codés comme inconnus. Un processus de validation des données a été conduit avec les provinces et territoires en mars 2015 pour tous les cas de rougeole déclarés sur l'ensemble du pays en 2014. 
La répartition des cas de rougeole selon les données démographiques, le statut vaccinal et les hospitalisations a été évaluée. Les analyses génotypiques et phylogénétiques ont également été décrites. Les nombres et les pourcentages de cas ont été déterminés pour les variables nominales, tandis que les valeurs moyennes et médianes ou les fourchettes ont été déterminées pour les variables continues. Les taux d'incidence ont été calculés à l'aide des estimations de la population de 2014 de Statistique Canada en date du $1^{\text {er }}$ juillet, en unités conformes aux indicateurs recommandés de l'OPS, soit $X$ cas pour 1000000 habitants (9). Le statut d'élimination a été évalué au moyen des critères établis par l'OPS (9).

Une éclosion était définie comme l'apparition de deux cas confirmés ou plus, liés sur le plan épidémiologique ou virologique, ou les deux (11). Le calendrier actuel de vaccination financé par l'État dans la province ou le territoire déclarant le cas a été utilisé pour définir si la couverture par le vaccin contenant le virus de la rougeole était à jour (15) au moment de l'apparition de l'éruption cutanée. Les cas âgés de moins d'un an et ceux nés avant 1970 n'étaient pas admissibles à recevoir le vaccin en raison de leur âge, et ont donc été classés parmi les sujets à jour pour leur âge, le statut vaccinal n'important pas. Les personnes âgées de sept ans ou plus, ou nées après 1970, étaient définies comme étant à jour si elles avaient reçu deux doses du vaccin. Finalement, pour les jeunes enfants âgés d'un à six ans, l'administration d'une ou de deux doses, selon le calendrier en vigueur dans la province ou le territoire de déclaration, les qualifiait parmi les sujets à jour. Des analyses épidémiologiques descriptives ont été réalisées au moyen du logiciel Enterprise Guide de SAS 5.1 (16). Étant donné que ce rapport utilise les données de surveillance de la santé publique, elles n'avaient pas à être approuvées par un comité d'examen éthique.

\section{Résultats}

\section{Aperçu}

En 2014, un total de 418 cas de rougeole ont été déclarés, parmi lesquels $29,2 \%$ ( $n=122$ ) ont été confirmés en laboratoire et $70,8 \%(n=296)$ jugés comme épidémiologiquement liés à un cas confirmé en laboratoire. L'incidence globale durant cette période a été de 11,8 cas de rougeole pour 1000000 habitants.

Confinés dans le temps, les cas sont survenus à l'intérieur d'une période de 36 semaines, soit de la semaine se terminant le 4 janvier 2014 (semaine 1) à la semaine se terminant le 6 septembre 2014 (semaine 36). Pendant 17 semaines continues, soit de la semaine 37 (se terminant le 13 septembre 2014) à la semaine 53 (se terminant le 3 janvier 2015), aucun cas de rougeole n'a été déclaré. Le nombre le plus élevé de cas déclarés durant une seule semaine épidémiologique a été de 138 (33,0 \%); ce sommet a été atteint lors d'une éclosion en ColombieBritannique survenue la semaine 12 (se terminant le 22 mars 2014) (figure 1).

Figure 1 : Répartition des cas déclarés de rougeole par semaine épidémiologique d'apparition de l'éruption cutanée et par province ou territoire, au Canada, en 2013

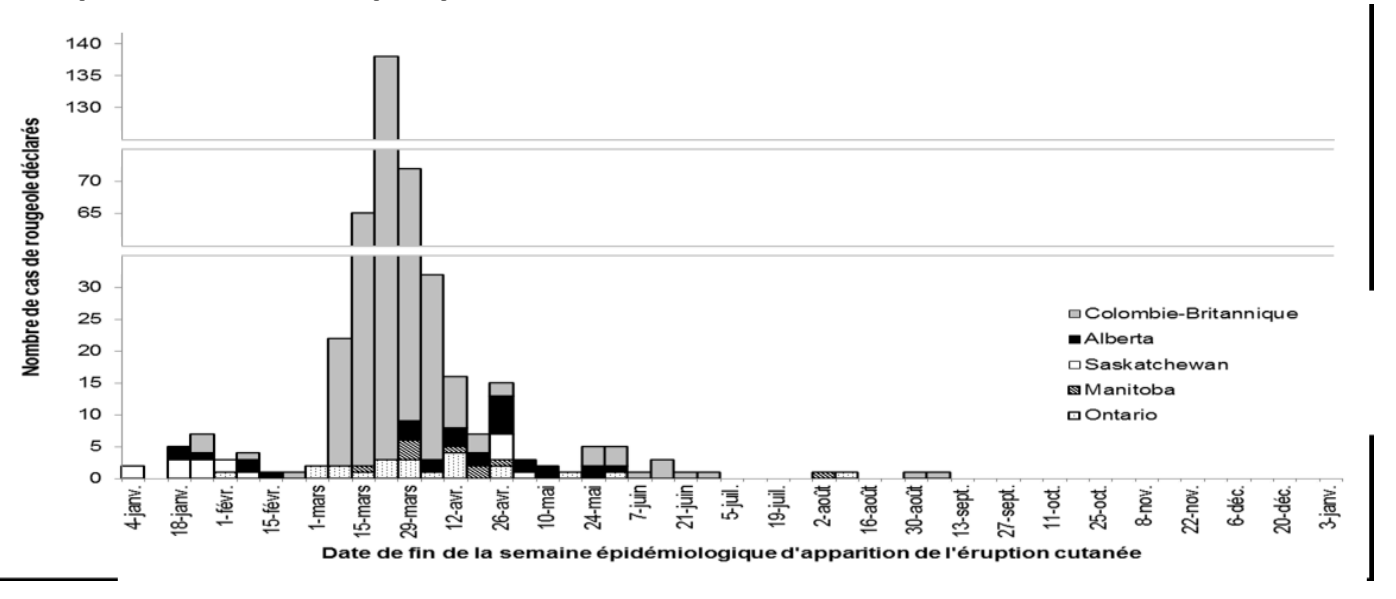


Répartition en fonction de l'âge, du sexeet de la zone géographique

Les cas les plus fréquents de rougeole ont été signalés chez les enfants âgés de cinq à neuf ans $(29,9 \%)$, suivis des jeunes adolescents âgés de 10 à 14 ans (25,4\%), puis des adolescents âgés de 15 à 19 ans (17,2\%)

(tableau 1).

Tableau 1 : Répartition des cas confirmés de rougeole et des taux d'incidence (pour 1000000 habitants) par groupe d'âge, sexe et province ou territoire de déclaration*, au Canada, en 2014

\begin{tabular}{|c|c|c|c|c|c|c|c|c|c|}
\hline Groupe d'âge & H & F & C.-B. & Alb. & Sask. & Man. & Ont. & Can. & $\begin{array}{c}\text { Taux global } \\
\text { d'incidence }\end{array}$ \\
\hline$<1$ an & 10 & 4 & $4^{\dagger}$ & 4 & 3 & 0 & 3 & 14 & 36,4 \\
\hline 1 à 4 ans & 13 & 12 & 17 & 3 & 2 & 1 & 2 & 25 & 16,2 \\
\hline 5 à 9 ans & 64 & 61 & 118 & 3 & 3 & 0 & 1 & 125 & 65,2 \\
\hline 10 à 14 ans & 56 & 50 & 100 & 3 & 2 & 0 & 1 & 106 & 56,8 \\
\hline 15 à 19 ans & 31 & 41 & 62 & 5 & 0 & 2 & 3 & 72 & 33,7 \\
\hline 20 à 24 ans & 15 & 12 & 23 & 2 & 0 & 1 & 1 & 27 & 10,9 \\
\hline 25 à 29 ans & 8 & 3 & 8 & 1 & 0 & 1 & 1 & 11 & 4,5 \\
\hline 30 à 39 ans & 13 & 11 & 7 & 4 & 5 & 1 & 7 & 24 & 5,0 \\
\hline 40 à 59 ans & 6 & 8 & 3 & 4 & 1 & 3 & 3 & 14 & 1,4 \\
\hline 60 ans et plus & 0 & 0 & 0 & 0 & 0 & 0 & 0 & 0 & - \\
\hline Total & $\mathbf{2 1 6}$ & $\mathbf{2 0 2}$ & $\mathbf{3 4 2}$ & $\mathbf{2 9}$ & $\mathbf{1 6}$ & $\mathbf{9}$ & $\mathbf{2 2}$ & $\mathbf{4 1 8}$ & \\
\hline Taux d'incidence : & 12,3 & 11,3 & 73,8 & 7,0 & 14,2 & 7,0 & 1,6 & 11,8 & 1,8 \\
\hline
\end{tabular}

* Les provinces et les territoires n'ayant déclaré aucun cas de rougeole au cours de 2014 ont été omis du tableau : Québec, Nouveau-Brunswick, NouvelleÉcosse, Île-du-Prince-Édouard, Terre-Neuve-et-Labrador, Yukon, Territoires du Nord-Ouest et Nunavut.

$\dagger$ Un cas de rougeole a été exclu de ce compte; il s'agissait d'un voyageur canadien qui a été exposé et infecté à l'extérieur du Canada et qui y est demeuré (à l'extérieur) pendant toute la durée de la maladie. Ce cas apparaît dans le bilan provincial de la Colombie-Britannique.

Les taux d'incidence les plus élevés ont été observés chez les enfants âgés de cinq à neuf ans, les préadolescents âgés de 10 à 14 ans et les nourrissons âgés de moins d'un an (65,2, 56,8 et 36,4 cas pour 1000000 habitants, respectivement).

Fait notable, 3,3\% $(n=14)$ des cas déclarés étaient des nourrissons trop jeunes (moins d'un an) pour avoir reçu la première dose du vaccin contenant le virus de la rougeole, et $1,7 \%(n=7)$ des cas étaient présumés posséder une immunité naturelle contre la rougeole, laquelle aurait été conférée par une infection antérieure (cas nés avant 1970). Environ la moitié $(51,7 \%)$ des cas de rougeole déclarés en 2014 étaient des hommes.

Les cas déclarés de rougeole provenaient de cinq provinces canadiennes, soit la Colombie-Britannique $(n=342)$, l'Alberta $(n=29)$, l'Ontario $(n=22)$, la Saskatchewan $(n=16)$ et le Manitoba $(n=9)$. L'incidence la plus élevée était en Colombie-Britannique, suivie de la Saskatchewan et de l'Alberta (73,8, 14,2 et 7,0 cas pour 1000000 habitants, respectivement).

\section{Immunisation et vaccins}

Parmi les 418 cas de rougeole déclarés au cours de 2014, 8,1\% $(n=34)$ étaient considérés comme ayant une couverture à jour en fonction de l'âge pour ce qui est du vaccin contenant le virus de la rougeole, au moment de l'apparition de l'éruption cutanée (tableau 2). II faut noter toutefois que 61,8\% ( $n=21)$ de ces cas n'étaient pas admissibles à recevoir le vaccin en raison de leur âge (nourrissons âgés de moins d'un an [n $=14]$ et adultes nés avant 1970 [ $n=7]$ ), selon les recommandations actuelles du Comité consultatif national de l'immunisation (CCNI) (17). Quels que soient leurs antécédents de vaccination, ils étaient classés comme ayant une couverture vaccinale à jour en fonction de leur âge. Par conséquent, seulement 3,1\% $(n=13)$ de l'ensemble des cas étaient admissibles à recevoir le vaccin contenant le virus de la rougeole et à être considérés comme ayant une couverture vaccinale à jour en fonction de l'âge au moment de l'infection. 
Tableau 2 : Statut vaccinal des cas de rougeole confirmés, par groupe d'âge et nombre de doses reçues, au Canada, en 2014

\begin{tabular}{|c|c|c|c|c|c|}
\hline & 0 dose & 1 dose & $\geq 2$ doses & $\begin{array}{c}\mathrm{N}^{\text {bre }} \text { de doses } \\
\text { inconnu }\end{array}$ & Total \\
\hline Groupe d'âge & $\begin{array}{c}\text { (vaccination à } \\
\text { jour avec } \\
\text { 0 dose) }\end{array}$ & $\begin{array}{c}\text { (vaccination à } \\
\text { jour avec } \\
1 \text { dose) }\end{array}$ & $\begin{array}{c}\text { (vaccination à } \\
\text { jour avec } \\
\geq 2 \text { doses) }\end{array}$ & $\begin{array}{l}\text { (vaccination à } \\
\text { jour avec un } \\
n^{\text {bre inconnu de }} \\
\text { dose) }\end{array}$ & $\begin{array}{c}\text { (vaccination à } \\
\text { jour) }\end{array}$ \\
\hline$<1$ an & $12(12)$ & $1(1)$ & $0(-)$ & 1 (1) & $14(14)$ \\
\hline 1 à 4 ans & $23(0)$ & $1(1)$ & $0(-)$ & $1(0)$ & $25(1)$ \\
\hline 5 à 9 ans & $105(0)$ & $0(-)$ & $0(-)$ & $20(0)$ & $125(0)$ \\
\hline 10 à 14 ans & $90(0)$ & $0(-)$ & $2(2)$ & $14(0)$ & $106(2)$ \\
\hline 15 à 19 ans & $61(0)$ & $2(0)$ & $4(4)$ & $5(0)$ & $72(4)$ \\
\hline 20 à 24 ans & $22(0)$ & $0(-)$ & $1(1)$ & $4(0)$ & 27 (1) \\
\hline 25 à 29 ans & $9(0)$ & $0(-)$ & 1 (1) & $1(0)$ & $11(1)$ \\
\hline 30 à 39 ans & $5(0)$ & $6(0)$ & $3(3)$ & $10(0)$ & $24(3)$ \\
\hline 40 à 59 ans & $3(2)$ & $0(-)$ & $1(1)$ & $10(5)$ & $14(8)$ \\
\hline 60 ans et plus & $0(-)$ & $0(-)$ & $0(-)$ & $0(-)$ & $0(-)$ \\
\hline TOTAL & 330 (14) & $10(2)$ & 12 (12) & $66(6)$ & 418 (34) \\
\hline
\end{tabular}

Selon les antécédents de vaccination, $91,9 \%(n=384)$ des cas n'étaient pas considérés comme ayant une vaccination à jour en fonction de l'âge, y compris $14,4 \%(n=60)$ de cas aux antécédents de vaccination inconnus qui ne pouvaient être évalués. Les cas dont la couverture par le vaccin contenant le virus de la rougeole n'était pas considérée à jour en fonction de l'âge étaient les plus nombreux dans le groupe des cinq à neuf ans $(32,6 \%)$, suivis des 10 à 14 ans $(27,1 \%)$, puis des 15 à 19 ans $(17,7 \%)$.

Un cas de rougeole a été signalé chez un enfant âgé de moins d'un an qui avait déjà reçu une dose du vaccin contenant le virus de la rougeole. Toutefois, le vaccin avait été administré peu de temps avant l'apparition de l'éruption cutanée, et le cas avait finalement été considéré comme non vacciné au moment de l'exposition.

Fait notable, trois cas ont été signalés chez des personnes ayant reçu trois doses du vaccin contenant le virus de la rougeole. Ces trois cas avaient été importés des Philippines et avaient reçu leur première dose du vaccin contenant le virus de la rougeole à l'extérieur du Canada avant l'âge de douze mois.

\section{Hospitalisation}

Au cours de 2014, 5,0 \% ( $n=21)$ des cas de rougeole ont présenté une indication d'hospitalisation (tableau 3).

Tableau 3 : Données d'hospitalisation des cas confirmés de rougeole, par groupe d'âge, au Canada, en 2013

\begin{tabular}{|c|c|c|c|c|}
\hline \multirow{2}{*}{ Groupe d'âge } & \multirow{2}{*}{ Total } & Non hospitalisé & Hospitalisé & $\mathbf{N}^{\text {bre }}$ de doses inconnu \\
\cline { 3 - 5 } & & $\mathrm{N}^{\text {bre }}(\%)$ & $\mathbf{N}^{\text {bre }}(\%)$ & $\mathbf{N}^{\text {bre }}(\%)$ \\
\hline$<1$ an & 14 & $7(50,0)$ & $5(35,7)$ & $2(14,3)$ \\
\hline 1 à 4 ans & 25 & $22(88,0)$ & $3(12,0)$ & $0(-)$ \\
\hline 5 à 9 ans & 125 & $124(99,2)$ & $1(0,8)$ & $0(-)$ \\
\hline 10 à 14 ans & 106 & $106(100,0)$ & $0(-)$ & $0(-)$ \\
\hline 15 à 19 ans & 72 & $72(100,0)$ & $0(-)$ & $0(-)$ \\
\hline 20 à 24 ans & 27 & $26(96,3)$ & $1(3,7)$ & $0(-)$ \\
\hline
\end{tabular}




\begin{tabular}{|c|c|c|c|c|}
\hline 25 à 29 ans & 11 & $10(90,9)$ & $1(9,1)$ & $0(-)$ \\
\hline 30 à 39 ans & 24 & $16(66,7)$ & $5(20,8)$ & $3(12,5)$ \\
\hline 40 à 59 ans & 14 & $8(57,1)$ & $5(35,7)$ & $1(7,1)$ \\
\hline 60 ans et plus & 0 & $0(-)$ & $0(-)$ & $0(-)$ \\
\hline Total & 418 & $391(93,5)$ & $21(5,0)$ & $6(1,4)$ \\
\hline
\end{tabular}

Les groupes d'âge le plus jeune et le plus vieux ont été confrontés au fardeau le plus lourd, quelque 35,7\% $(n=5)$ des cas âgés de moins d'un an, 35,7\% $(n=5)$ des cas âgés de 40 à 59 ans et 20,8 \% $(n=5)$ des cas âgés de 30 à 39 ans ayant dû être hospitalisés. Les hospitalisations déclarées chez les cas âgés de 1 à 29 ans étaient peu fréquentes.

Il est à noter que le statut d'hospitalisation n'était pas connu chez $1,4 \%(n=6)$ des cas.

\section{Épidémiologie moléculaire}

En 2014, les échantillons disponibles ont permis de déterminer le génotype de $26,6 \%(n=111)$ des cas déclarés de rougeole. Les cas ayant un lien épidémiologique avec un cas génotypé présentent habituellement le même génotype, c'est pourquoi, aux fins de surveillance, il n'est pas nécessaire d'obtenir le génotype de tous les cas d'une éclosion. Ainsi, en 2014, on a réussi à obtenir le génotype de $98 \%$ des événements rougeoleux uniques, où chaque éclosion ou cas sporadique était considéré comme un seul événement (18 éclosions sur 18, et 31 cas sporadiques sur 32) (tableau 4). Les génotypes de la rougeole étaient les suivants : B3 $(n=65)$, D8 $(n=36)$, D9 $(n=5), H 1(n=3)$ et D4 $(n=2)$ (figure 2).

Figure 2 : Répartition des génotypes des souches de rougeole détectées dans les cas de 2014 ( $n=111$ ) par semaine d'apparition de l'éruption cutanée (si disponible, $n=94$ ) ou collecte d'échantillons ( $n=17)$

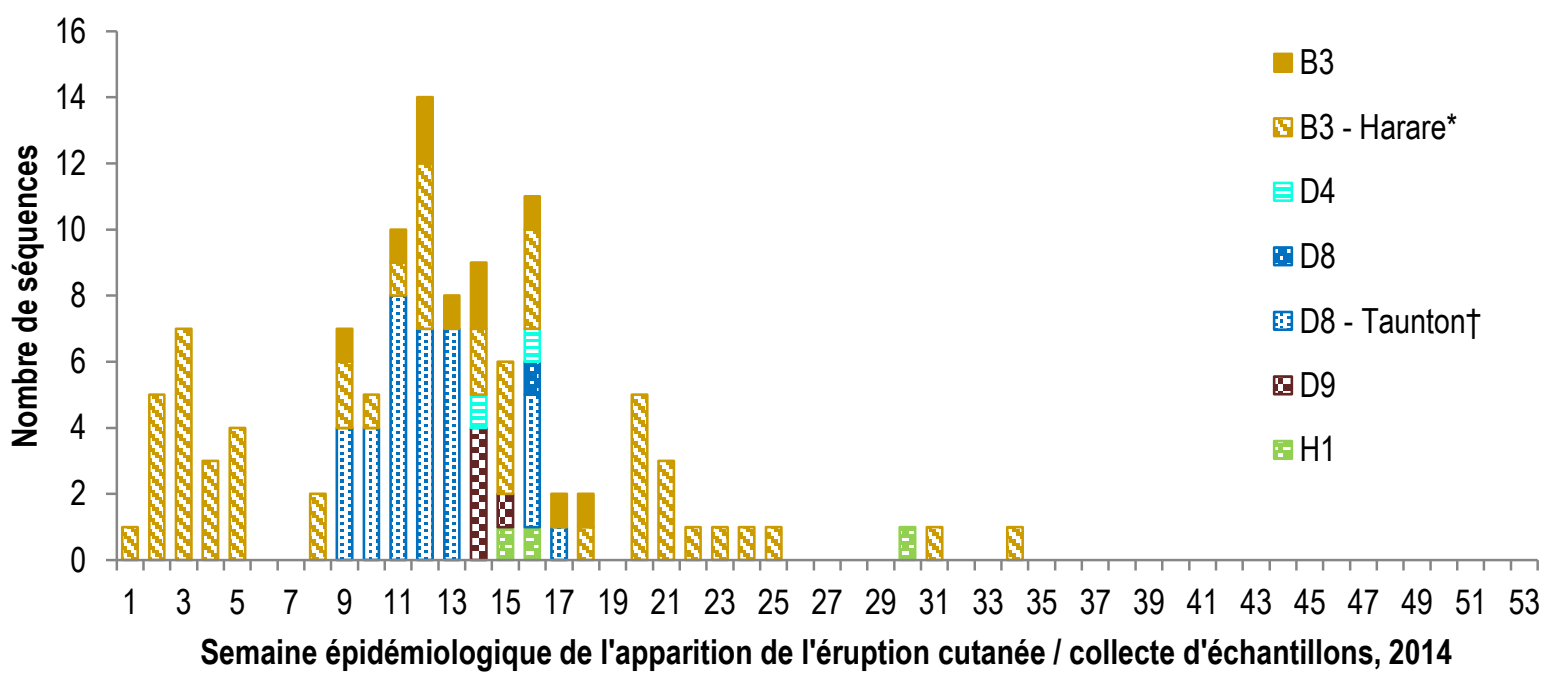

Les semaines épidémiologiques sont attribuées conformément aux lignes directrices de l'Organisation mondiale de la Santé (WHO.WER.2012;9(87):73), avec la semaine 1 commençant le premier lundi de chaque année.

* Séquences du génotype B3 identiques à la variante de séquence MVi/Harare.ZWE/38.09 (numéro d'enregistrement GenBank : JF973033).

$\dagger$ Séquences du génotype D8 identiques à la variante de séquence MVs/Taunton.GBR/27.12 (numéro d'enregistrement GenBank: JX984461).

En 2014, le génotype B3 de la rougeole a circulé partout dans le monde : il a été déclaré dans plus de 40 pays (18). Au Canada, il a été détecté chez 65 cas, dont 58,5\% $(n=38)$ étaient associés à 12 éclosions (annexe). Ce génotype a été le plus souvent importé des Philippines $(n=16)$, mais a aussi été relevé chez des cas ayant 
voyagé aux États-Unis ( $n=1)$, en Europe $(n=1)$, au Pakistan $(n=1)$ et en Thaïlande $(n=1)$. Cinquante-cinq des 65 séquences $\mathrm{N}-450$ du génotype 3 étaient identiques à la variante de séquence MVi/Harare.ZWE/38.09 (numéro d'enregistrement GenBank JF973033), aussi connue comme la variante de séquence B3-Harare (figure 3). Cette variante de séquence a été déclarée partout dans le monde et a été fréquemment liée aux Philippines, où une importante éclosion est survenue en 2014 (numéro d'enregistrement GenBank KJ634500). Tous les cas canadiens ayant voyagé aux Philippines présentaient des séquences $\mathrm{N}-450$ identiques à la variante de séquence B3-Harare $(n=13)$ ou ne différant que par un nucléotide $(99,8 \%$ identique, $n=3)$. Les variantes de séquence du génotype B3 importées de pays autres que les Philippines ne différaient de la variante de séquence B3-Harare que par un $(99,8 \%$ identique, importée des États-Unis, $n=1)$, deux $(99,6 \%$ identique, importée de Thaïlande, $n=1$ ) ou trois nucléotides ( $99,3 \%$ identique, importée du Pakistan, $n=1)$, la confirmation en laboratoire fournissant ainsi la preuve que ces événements rougeoleux n'étaient pas liés, ayant été importés de multiples sources.

Figure 3 : Arbre phylogénétique des séquences rougeoleuses N-450 détectées au Canada en 2014 $(n=111)$

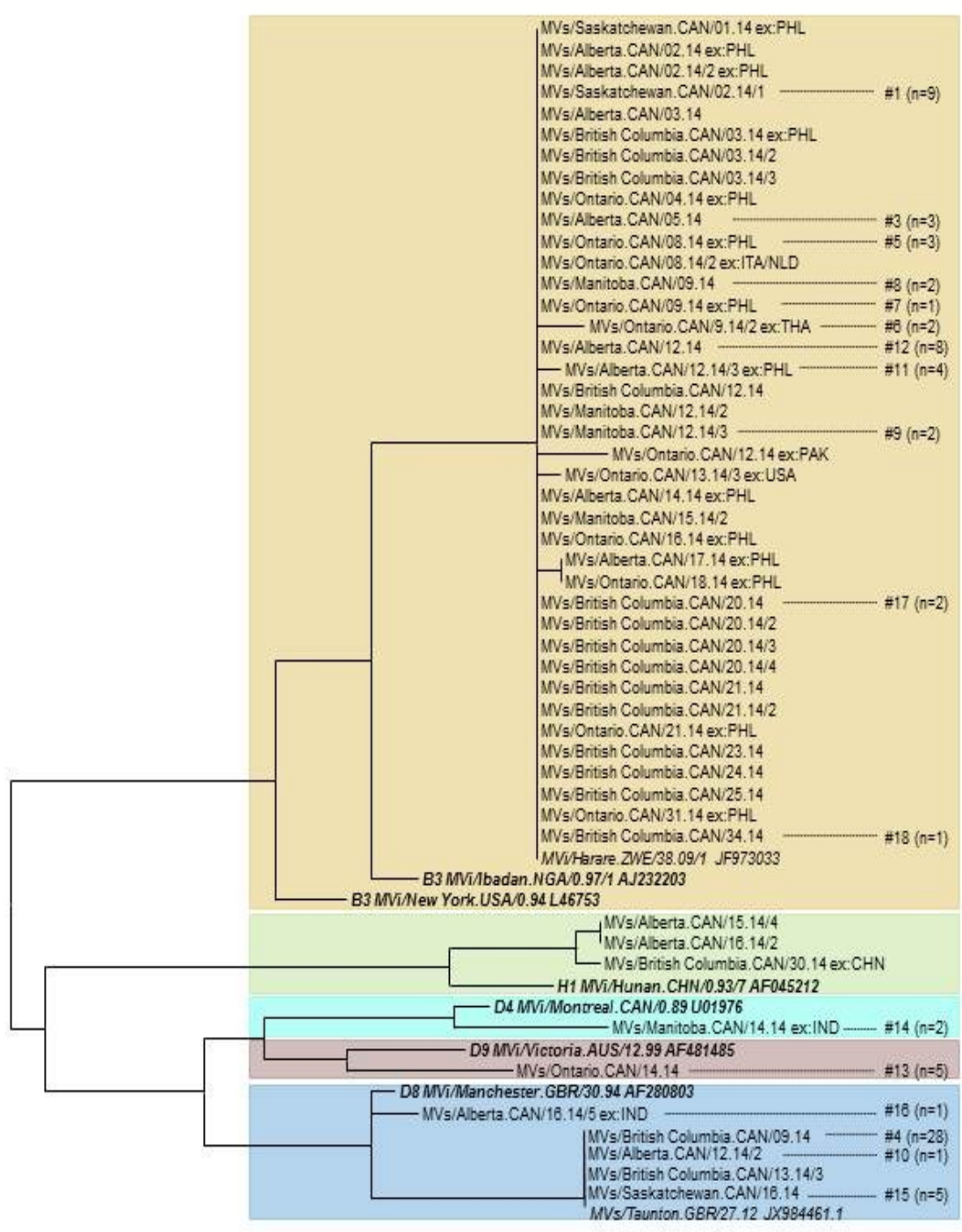




\section{LÉGENDE :}

Les séquences de référence pertinentes de l'OMS (13) sont indiquées en caractères gras italiques. Les variantes de séquence, assignées dans la base de données des séquences de la rougeole de l'OMS (MeaNS) (20), sont indiquées en italiques. Les séquences canadiennes sont représentées en caractères ordinaires et sont identifiées par la désignation de l'OMS qui indique la province et la semaine d'apparition de l'éruption cutanée ou de la collecte de l'échantillon. Les cas ayant voyagé sont identifiés par « ex: » suivi des trois lettres du code du pays. Les éclosions sont représentées par une seule séquence et sont identifiées par leur numéro d'éclosion (annexe). Le nombre de séquences identiques relevées dans l'éclosion est fourni entre parenthèses. Les séquences restantes, n'ayant aucun numéro d'éclosion, proviennent de cas sporadiques $(\mathrm{n}=31)$.

Le génotype D8 de la rougeole a été détecté dans 36 cas, parmi lesquels $88,9 \%(n=32)$ étaient associés à quatre éclosions, y compris celle de grande ampleur en Colombie-Britannique (annexe). Presque toutes les séquences $\mathrm{N}-450(\mathrm{n}=35)$ (dont bon nombre étaient associées à trois éclosions) étaient identiques à la variante de séquence MVs/Taunton.GBR/27.12 (numéro d'enregistrement GenBank JX984461) (figure 3). À l'échelle mondiale, cette variante de séquence du génotype $\mathrm{D} 8$ a déjà été associée à une importante et longue éclosion aux Pays-Bas ayant débuté en 2013 (19). Une deuxième souche du génotype D8 a été identifiée en 2014, laquelle a été associée à une petite éclosion ayant pris naissance en Inde, pays où le génotype D8 est endémique (20) (figure 3, annexe).

Les éclosions déclarées étaient de taille variable ( 2 à 325 cas; médiane : 3 cas). La majorité $(72,2 \%)$ des éclosions avaient deux générations ou moins, tandis que leur durée médiane était de 15 jours (deux générations). Le génotype des éclosions déclarées étaient B3 $(n=12)$, D8 $(n=4)$, D4 $(n=1)$ et D9 $(n=1)$.

La grande majorité $(84,4 \%, n=325)$ des cas liés à une éclosion sont survenus au cours d'une importante éclosion de rougeole en Colombie-Britannique. Cette éclosion s'est produite au sein d'une communauté religieuse ne pratiquant pas la vaccination. On soupçonne que la source du virus était une importation non documentée des Pays-Bas, pays où sévissait une éclosion concomitante (19) au sein d'une communauté religieuse ayant des antécédents d'importation de maladies évitables par la vaccination au Canada $(1,21,22)$. Cette éclosion a touché principalement des enfants et des adolescents âgés de 5 à 14 ans. L'analyse détaillée de cette éclosion est décrite ailleurs (8).

\section{La rougeole canadienne dans le contexte mondial}

Parmi les 418 cas de rougeole déclarés en 2014, 6,0\% $(n=25)$ ont été importés. La majorité des importations ont contracté la maladie dans la région Pacifique occidental (Philippines [ $n=18]$ et Chine $[n=1]$ ), suivie des régions Asie du Sud-Est (Inde [ $n=2]$ et Thaïlande $[n=1]$ ), Méditerranée orientale (Pakistan $[n=1]$ ), Europe (Italie/Pays-Bas [ $n=1]$ ) et Amériques (États-Unis [n $=1]$ ). Les Philippines ont été victimes d'une importante éclosion de rougeole en 2014, laquelle a sans doute été à l'origine des nombreuses importations observées au Canada cette année-là.

Parmi les 25 importations déclarées, seulement neuf (en provenance des Philippines [ $n=6$ ], de l'Inde [ $n=2$ ] et de la Thaïlande $[n=1]$ ) ont, selon les connaissances actuelles, entraîné une propagation secondaire. Toutes les importations déclarées étaient des individus dont le pays de résidence habituelle était le Canada; ils ont tous contracté la rougeole lors d'un voyage et ils étaient tous contagieux à leur retour au Canada. En outre, les importations étaient réparties dans presque tous les groupes d'âge, bien que plus fréquemment dans les groupes d'âge des moins d'un an et des 15 à 19 ans ( $16 \%, n=4$, chaque groupe).

\section{Maintien de l'élimination de la rougeole}

L'OPS a établi quatre critères essentiels pour la vérification continue de l'élimination de la rougeole (9). Le Canada a satisfait pleinement ou partiellement à tous ces critères en 2014 (tableau 4). 
Tableau 4 : Critères essentiels de l'Organisation panaméricaine de la santé pour la vérification de I'élimination de la rougeole

\begin{tabular}{|c|c|c|}
\hline Critère & Indicateur & Description \\
\hline $\begin{array}{l}\text { Vérifier l'interruption des cas } \\
\text { endémiques de rougeole au } \\
\text { cours d'une période d'au moins } \\
\text { trois ans à compter du dernier } \\
\text { cas endémique connu, en } \\
\text { présence d'une surveillance de } \\
\text { qualité supérieure }\end{array}$ & $\begin{array}{l}\text { Aucun cas de transmission } \\
\text { endémique }\end{array}$ & $\begin{array}{l}\text { Critère satisfait } \\
\text { Le Canada est parvenu à éradiquer la rougeole en } 1998 . \\
\text { Depuis lors, la surveillance moléculaire et } \\
\text { épidémiologique continue de démontrer l'absence de } \\
\text { souche virale en circulation pendant un an ou plus au } \\
\text { Canada. } \\
\text { Les données précédemment présentées à l'appui de la } \\
\text { vérification de l'interruption de la rougeole endémique au } \\
\text { Canada de } 1998 \text { à } 2013 \text { sont disponibles en ligne }(1,2 \text {, } \\
\text { 3). }\end{array}$ \\
\hline $\begin{array}{l}\text { Maintenir une surveillance de } \\
\text { qualité supérieure suffisamment } \\
\text { sensible pour déceler les cas } \\
\text { importés et ceux liés à } \\
\text { l'importation }\end{array}$ & $\begin{array}{l}>2 \text { cas suspectés pour } \\
100000 \text { habitants ont fait } \\
\text { l'objet d'une enquête } \\
\text { adéquate }\end{array}$ & $\begin{array}{l}\text { Critère partiellement satisfait } \\
\text { Étant donné que seuls les cas confirmés de rougeole } \\
\text { doivent obligatoirement être déclarés au Canada, cet } \\
\text { indicateur ne peut être évalué directement. } \\
\text { Toutefois, grâce aux données du projet pilote sur la } \\
\text { surveillance de la rougeole et de la rubéole, on a estimé } \\
\text { le taux national d'enquêtes sur les maladies évoquant la } \\
\text { rougeole, lequel s'est situé entre } 12 \text { pour } \\
100000 \text { habitants (2006, année sans éclosion) et } 19 \text { pour } \\
100000 \text { habitants (2011, année touchée par une } \\
\text { éclosion). }\end{array}$ \\
\hline $\begin{array}{l}\text { Vérifier l'absence de souches de } \\
\text { virus endémiques de la rougeole } \\
\text { en assurant une surveillance } \\
\text { virologique }\end{array}$ & $\begin{array}{l}\text { Génotypage de la rougeole } \\
\text { effectué lors de } 80 \% \text { des } \\
\text { éclosions }\end{array}$ & $\begin{array}{l}\text { Critère satisfait } \\
\text { On a obtenu le génotype d'au moins un cas lors de } \\
\text { chacune des éclosions de } 2014 \text {. }\end{array}$ \\
\hline
\end{tabular}

\section{Discussion}

En 2014, 418 cas confirmés de rougeole ont été déclarés au Canada, ce qui représente le deuxième plus haut total depuis l'éradication de la rougeole en 1998. Ces cas étaient cependant en grande partie attribuables à une éclosion survenue dans une communauté religieuse ne pratiquant pas la vaccination (8). Fait notable, la majorité $(72,2 \%)$ des éclosions en 2014 étaient limitées à deux générations ou moins, tandis que leur durée médiane était de 15 jours (deux générations). Cela donne à penser que ces événements étaient bien contrôlés par la combinaison de taux de couverture vaccinale élevés et d'interventions mises en œuvre par la santé publique. 
Le fardeau de la maladie (incidence de la rougeole) a été le plus élevé chez les enfants et les adolescents âgés de 5 à 14 ans, ce qui correspond à la répartition par groupe d'âge des cas déclarés lors d'une éclosion survenue en Colombie-Britannique dans une communauté religieuse ne pratiquant pas la vaccination. En revanche, le fardeau lié à la morbidité (c'est-à-dire, les hospitalisations) a tout de même été appréciable chez les moins d'un an et les 40 à 59 ans. Compte tenu des limites associées aux données sur la morbidité saisies dans le SCSRR et le projet pilote sur la surveillance de la rougeole et de la rubéole, et de l'absence de données sur la mortalité, il s'agit d'une représentation incomplète du fardeau réel de la maladie causée par la rougeole au Canada.

Cinq génotypes (B3, D8, D9, H1 et D4) ont été détectés en 2014, lesquels ont été importés de toutes les régions de l'OMS sauf l'Afrique. Même si le Canada a réussi à obtenir le statut d'élimination de la rougeole en 1998, les importations de cas de rougeole au pays se poursuivront tant et aussi longtemps que le virus circule libéralement dans les autres pays. Selon l'OMS, plus de 201000 cas confirmés de rougeole ont été déclarés dans le monde en 2014, principalement dans la région du Pacifique occidental (23). Aussi, le total annuel de cas de rougeole déclarés aux États-Unis était le plus élevé depuis leur obtention du statut d'élimination du virus en 2000 (24). Les voyages internationaux, combinés aux éclosions importantes dans d'autres régions du monde, peuvent accroître le risque d'importation et, subséquemment, de propagation de la rougeole au Canada. Les grandes éclosions au pays, telle celle signalée en Colombie-Britannique, font ressortir les points à améliorer dans l'immunité de la population, ainsi que l'importance continue de maintenir une couverture élevée par le vaccin contenant le virus de la rougeole.

Comme les années précédentes, le Canada a pleinement ou partiellement satisfait à tous les critères de vérification et d'élimination énoncés par l'OPS. On a réussi à obtenir le génotype de $98 \%$ des événements rougeoleux uniques en 2014. Les méthodes moléculaires continuent à jouer un rôle clé dans la surveillance épidémiologique liée aux critères suivants ayant été pleinement satisfaits par le Canada en 2014 : « Vérifier l'interruption des cas endémiques de rougeole au cours d'une période d'au moins trois ans à compter du dernier cas endémique connu, en présence d'une surveillance de qualité supérieure » et « Vérifier l'absence de souches de virus endémiques de la rougeole en assurant une surveillance virologique ». Les critères partiellement satisfaits par le Canada, soit « maintenir une surveillance de qualité supérieure suffisamment sensible pour déceler les cas importés et ceux liés à l'importation » et « veiller à ce que l'immunisation au sein de la population soit adéquate », fournissent l'occasion de renforcer les activités actuelles liées à l'engagement continu du Canada envers l'élimination de la rougeole, telles que résumées ailleurs (25).

\section{Limites}

Les décès liés à la rougeole ne sont pas actuellement saisis dans le SCSRR ou le projet pilote sur la surveillance de la rougeole et de la rubéole. Toutefois, il est probable que les décès attribuables à une infection aiguë par le virus de la rougeole seraient décrits dans d'autres sources d'information (p. ex. Statistique Canada, statistiques de l'état civil ou médias). Bien que certaines données limitées sur la morbidité soient disponibles, aucun renseignement détaillé, telle la durée de l'hospitalisation ou la nature et la gravité des complications, n'est actuellement décrit dans le SCSRR ou le projet pilote sur la surveillance de la rougeole et de la rubéole.

Le statut vaccinal n'a pu être évalué dans $14,4 \%(n=60)$ des cas. En outre, le statut vaccinal des cas âgés d'un à six ans était défini au moyen du calendrier en vigueur dans la province ou le territoire, lequel peut varier d'une administration à l'autre. Bien que le cadre de santé dans lequel survient le cas soit respecté, une distinction peut se produire entre deux individus semblables, laquelle est fondée sur un facteur qui pourrait ne pas contribuer à leur vulnérabilité à la rougeole.

\section{Conclusion}

Malgré une activité importante de la rougeole en 2014, le Canada continue de fournir de solides preuves que son statut d'élimination de la rougeole est maintenu. 


\section{Remerciements}

Les auteurs souhaitent remercier leurs partenaires provinciaux et territoriaux pour leur collaboration et leurs efforts continus à fournir et à valider les données saisies dans le SCSRR et le projet pilote sur la surveillance de la rougeole et la rubéole, ainsi que pour leur revue du contenu de ce rapport.

Les auteurs sont également reconnaissants envers le personnel du LNM, en particulier celui des divisions Services principaux de la génomique et Exanthèmes viraux ( $D^{\text {rs }}$ Alberto Severini, Lillian Mendoza et Lisa Podhorodecki) pour leur contribution dans la production des données moléculaires, et les laboratoires provinciaux pour remise d'échantillons.

\section{Conflit d'intérêts}

Aucun

\section{Financement}

Ce travail a été appuyé par l'Agence de la santé publique du Canada.

\section{Références}

(1) King A, Varughese P, De Serres G, Tipples GA, Waters J. Working Group on Measles Elimination. Measles elimination in Canada. J Infect Dis. 2004 May 1;189 Suppl 1:S236-42.

(2) Public Health Agency of Canada. Elimination of measles, rubella and congenital rubella syndrome in Canada: Documentation and verification report. Ottawa ON: Public Health Agency of Canada; 2011.

Disponible en français: Agence de la santé publique du Canada. Élimination de la rougeole, de la rubéole et du syndrome de rubéole congénitale au Canada. Documentation et rapport de vérification. 2011.

(3) Shane A, Hiebert J, Sherrard L, Deehan H. Measles surveillance in Canada: Trends for 2013. CCDR. 2014;40(12):219.

Disponible en français : http://www.phac-aspc.gc.ca/publicat/ccdr-rmtc/14vol40/dr-rm40-12/dr-rm40-12-surv-1-fra.php

(4) World Health Organization (WHO). Monitoring progress towards measles elimination. Wkly Epidemiol Rec. 2010 Dec 3;85(49):490-4).

http://www.who.int/wer/2010/wer8549.pdf.

(5) Hiebert J, Severini A. Measles molecular epidemiology: What does it tell us and why is it important? CCDR. 2014;40(12):257.

Disponible en français : http://www.phac-aspc.gc.ca/publicat/ccdr-rmtc/14vol40/dr-rm40-12/dr-rm40-12-over-fra.php.

(6) Castillo-Solorzano C, Matus C, Flannery B, Marsigli C, Tambini G, Andrus J. The Americas: Paving the road toward global measles eradication. J Infect Dis. 2011;204(Suppl 1):S270.

(7) Public Health Agency of Canada. Vaccine coverage in Canadian children: Results from the 2011 Childhood National Immunization Coverage Survey. Ottawa ON: Public Health Agency of Canada; 2014. http://www.phac-aspc.gc.ca/im/nics-enva/vccc-cvec-eng.php. Disponible en français : http://www.phac-aspc.gc.ca/im/nics-enva/vccc-cvec-fra.php.

(8) Pan American Health Organization (PAHO). Plan of action. Documentation and verification of measles, rubella and congenital rubella syndrome elimintation in the Region of the Americas. Technical Document. Washington DC: PAHO; 2010.

(9) Naus M, Puddicombe D, Murti M, Fund C et al. Outbreak of measles in an unvaccinated population, British Columbia, 2014. CCDR. 2015; 41:7 (171-177).

(10) Public Health Agency of Canada. Case definitions for diseases under national surveillance. CCDR. 2009;35(S2). http://www.phac-aspc.gc.ca/publicat/ccdr-rmtc/09vol35/35s2/index-eng.php.

Disponible en français : http://www.phac-aspc.gc.ca/publicat/ccdr-rmtc/09vol35/35s2/index-fra.php.

(11) Public Health Agency of Canada. Guidelines for the prevention and control of measles outbreaks in Canada. CCDR. 2013;39:ACS3.

Disponible en français : http://www.phac-aspc.gc.ca/publicat/ccdr-rmtc/13vol39/acs-dcc-3/index-fra.php

(12) World Health Organization (WHO). Standardization of the nomenclature for describing the genetic characteristics of wild-type measles viruses. Wkly Epidemiol Rec. 1998;73:265.

(13) World Health Organization (WHO). Measles virus nomenclature update: 2012. Wkly Epidemiol Rec. $2012 ; 9(87): 73$.

(14) Tamura K, Peterson D, Peterson N, Stecher G, Nei M, Kumar S. Molecular evolutionary genetics analysis using maximum likelihood, evolutionary distance, and maximum parsimony methods. Mol Biol Evol. 2011;28:2731.

(15) Public Health Agency of Canada, Canadian Nurses Coalition on Immunization.

Publicly-funded immunization programs in Canada: Routine schedule for infants and children including special programs and catch-up programs. Ottawa ON: Public Health Agency of Canada; 2015. 
http://www.phac-aspc.gc.ca/im/ptimprog-progimpt/table-1-eng.php.

Disponible en français : http://www.phac-aspc.gc.ca/im/ptimprog-progimpt/table-1-fra.php.

(16) SAS Global Forum 2014. SAS Enterprise Guide 5.1. 2013;5.1.

(17) National Advisory Committee on Immunization (NACI). Canadian immunization guide. Part 4: Active vaccines measles vaccine. Ottawa ON: Public Health Agency of Canada; 2012.

http://www.phac-aspc.gc.ca/publicat/cig-gci/p04-meas-roug-eng.php.

Disponible en français : http://www.phac-aspc.gc.ca/publicat/cig-gci/p04-meas-roug-fra.php.

(18) World Health Organization (WHO) [Internet]. Measles surveillance data. Geneva: World Health Organization; 2015. http://www.who.int/immunization/monitoring_surveillance/burden/vpd/surveillance_type/active/measles_monthlydata/ en/index1.html.

(19) Knol M, Urbanus A, Swart E, Mollema L, Ruijs W, van Vinnendijk R, et al. Large ongoing measles outbreak in a religious community in the Netherlands since May 2013. Euro Surveill. 2013 Sep 5;18(36).

(20) Rota PA, Brown K, Mankertz A, et al. Global distribution of measles genotypes and measles molecular epidemiology. J Infec Dis. 2011;204(Suppl1):8514.

(21) Hahné S, Macey J, van Binnendijk R, Kohl R, Dolman S, van der Veen Y, et al. Rubella outbreak in the Netherlands, 2004-2005: High burden of congenital infection and spread to Canada. Pediatr Infect Dis J. 2009 Sep;28(9):795-800.

(22) Wielders C, van Binnendijk R, Snijders B, Tipples GA, Cremer J, Fanoy E, et al. Mumps epidemic in orthodox religious low-vaccination communities in the Netherlands and Canada, 2007 to 2009. Euro Surveill. 2011 Oct 13;16(41).

(23) World Health Organization (WHO) WHO Measles Surveillance Data. Geneva: WHO; 2015.

http://www.who.int/immunization/monitoring_surveillance/burden/vpd/surveillance_type/active/measlesregionalsumm ary.pdf?ua=1.

(24) Gastanaduy P, Redd SB, Parker Fiebelkorn A, Rota JS, Rota PA, Bellini WJ, et al. Measles - United States, January 1 - May 23, 2014. MMWR. 2014 Jun 6;63(22):496.

(25) Lam P-P, Williams L, Gadient S, Squires S, St-Laurent M. Maintaining measles elimination in Canada: Moving forward. CCDR. 2015; 41:7 (178-181).

\section{Annexe: Sommaire des éclosions de rougeole au Canada, classées par date d'apparition de la première éruption cutanée, en 2014}

\begin{tabular}{|c|c|c|c|c|c|}
\hline $\mathbf{N}^{\circ}$ & Prov. & $\begin{array}{l}N^{\text {bre }} \\
\text { de } \\
\text { cas }\end{array}$ & $\begin{array}{c}\text { Durée (en jours) } \\
\text { (générations) }\end{array}$ & Génotype & Description \\
\hline 1 & Sask. & 10 & $\begin{array}{l}32 \\
(3)\end{array}$ & B3 - Harare & $\begin{array}{l}\text { - Le cas index était une importation des Philippines; il } \\
\text { s'agissait d'un voyageur canadien non immunisé. } \\
\text { - L'infection s'est ensuite propagée parmi les contacts } \\
\text { dans divers contextes. }\end{array}$ \\
\hline 2 & C.-B. & 2 & $\begin{array}{l}14 \\
(2)\end{array}$ & B3 - Harare* & $\begin{array}{l}\text { - La source d'exposition du cas index de cette éclosion } \\
\text { était inconnue. } \\
\text { - Le cas secondaire était épidémiologiquement lié au } \\
\text { cas index. }\end{array}$ \\
\hline 3 & Alb. & 3 & $\begin{array}{c}4 \\
(1)\end{array}$ & B3 - Harare* & $\begin{array}{l}\text { - Le cas primaire n'a pas été trouvé. } \\
\text { - Cette grappe de trois cas était présumée avoir } \\
\text { partagé une source commune d'exposition, selon les } \\
\text { dates d'apparition de l'éruption cutanée. }\end{array}$ \\
\hline 4 & C.-B. & 325 & $\begin{array}{l}107 \\
(9)\end{array}$ & D8 - Taunton $†$ & $\begin{array}{l}\text { - } \quad \text { Aucun cas primaire n'a été déclaré, mais on croit } \\
\text { qu'il pourrait s'agir d'une importation des Pays-Bas. } \\
\text { - L'infection s'est ensuite propagée au sein d'une } \\
\text { communauté religieuse ne pratiquant pas la } \\
\text { vaccination, principalement en milieu scolaire. } \\
\text { - Très peu de cas liés à cette éclosion ont été déclarés } \\
\text { à l'extérieur de la communauté religieuse. }\end{array}$ \\
\hline 5 & Ont. & 4 & $\begin{array}{l}26 \\
(3)\end{array}$ & B3 - Harare* & $\begin{array}{l}\text { - Le cas index de cette éclosion était une importation } \\
\text { des Philippines; il s'agissait d'un enfant non } \\
\text { immunisé. } \\
\text { - Les cas secondaires étaient des contacts à la maison } \\
\text { et à l'école. }\end{array}$ \\
\hline 6 & Ont. & 2 & $\begin{array}{l}12 \\
(2)\end{array}$ & B3 & $\begin{array}{l}\text { - Le cas index de cette éclosion était une importation } \\
\text { de la Thaïlande; il s'agissait d'un voyageur canadien }\end{array}$ \\
\hline
\end{tabular}




\begin{tabular}{|c|c|c|c|c|c|}
\hline & & & & & $\begin{array}{l}\text { déjà immunisé. } \\
\text { - Le cas secondaire était un contact familial du cas } \\
\text { index. }\end{array}$ \\
\hline 7 & Ont. & 3 & $\begin{array}{l}19 \\
(2)\end{array}$ & B3 - Harare* & $\begin{array}{l}\text { - Le cas index de cette éclosion était une importation } \\
\text { des Philippines, qui avait également voyagé en } \\
\text { Chine. } \\
\text { - Les deux cas secondaires ont été exposés à la } \\
\text { rougeole dans un établissement de soins de santé. }\end{array}$ \\
\hline 8 & Man. & 2 & $\begin{array}{l}15 \\
(2)\end{array}$ & B3 - Harare* & $\begin{array}{l}\text { - La source d'exposition du cas index de cette éclosion } \\
\text { était inconnue. } \\
\text { - Le cas secondaire était un contact familial proche du } \\
\text { cas index. }\end{array}$ \\
\hline 9 & Man. & 2 & $\begin{array}{l}21 \\
(2)\end{array}$ & B3 - Harare* & $\begin{array}{l}\text { - La source d'exposition du cas index de cette éclosion } \\
\text { était inconnue. } \\
\text { - L'exposition du cas secondaire a eu lieu dans un } \\
\text { établissement de soins de santé. }\end{array}$ \\
\hline 10 & Alb. & 2 & $\begin{array}{c}5 \\
(1)\end{array}$ & D8 - Taunton† & $\begin{array}{l}\text { - Le cas primaire de cette éclosion n'a pas été trouvé. } \\
\text { - Les deux cas déclarés étaient épidémiologiquement } \\
\text { liés et étaient présumés avoir une source commune } \\
\text { d'exposition. }\end{array}$ \\
\hline 11 & Alb. & 4 & $\begin{array}{l}27 \\
(3)\end{array}$ & B3 & $\begin{array}{l}\text { - Le cas index était une importation des Philippines; il } \\
\text { - L'agissait d'un Canadien non immunisé. } \\
\text { ees cas secondaires, associés à l'importation, étaient } \\
\text { épidémiologiquement liés et se trouvaient dans la } \\
\text { même unité de santé. }\end{array}$ \\
\hline 12 & Alb. & 9 & $\begin{array}{l}53 \\
(5)\end{array}$ & B3 - Harare* & $\begin{array}{l}\text { - Cet événement comprend deux importations } \\
\text { simultanées des Philippines (immigrants récents } \\
\text { ayant voyagé ensemble). } \\
\text { - Les cas secondaires étaient liés à l'importation. }\end{array}$ \\
\hline 13 & Ont. & 5 & $\begin{array}{c}12 \\
(1)\end{array}$ & D9 & $\begin{array}{l}\text { - Le cas index était un visiteur de la Chine } \\
\text { épidémiologiquement lié à une éclosion en cours } \\
\text { dans son pays. } \\
\text { - L'ensemble des cinq cas étaient } \\
\text { épidémiologiquement liés à ce visiteur. }\end{array}$ \\
\hline 14 & Man. & 2 & $\begin{array}{l}13 \\
(2)\end{array}$ & D4 & $\begin{array}{l}\text { - Le cas index de cette éclosion était importé de } \\
\text { l'Inde. } \\
\text { - Le cas secondaire présentait un faible lien } \\
\text { épidémiologique avec le cas index. }\end{array}$ \\
\hline 15 & Sask. & 5 & $\begin{array}{c}9 \\
(2)\end{array}$ & D8 - Taunton† & $\begin{array}{l}\text { - Le cas primaire lié à cet événement n'a pas été } \\
\text { identifié, mais on présume qu'il a été exposé à la } \\
\text { même source que les cas déclarés. } \\
\text { - Les cas déclarés sont regroupés en deux grappes, } \\
\text { mais aucune source d'exposition ni voyage n'a été } \\
\text { documenté. }\end{array}$ \\
\hline 16 & Alb. & 2 & $\begin{array}{l}13 \\
(2)\end{array}$ & D8 & $\begin{array}{l}\text { - Le cas index de cette éclosion était une importation } \\
\text { de l'Inde. } \\
\text { - Le cas secondaire était épidémiologiquement lié au } \\
\text { cas index. }\end{array}$ \\
\hline 17 & C.-B. & 2 & $\begin{array}{l}18 \\
(2)\end{array}$ & B3 - Harare* & $\begin{array}{l}\text { - La source d'exposition du cas index de cette éclosion } \\
\text { était inconnue. } \\
\text { - L'exposition du cas secondaire a eu lieu dans un } \\
\text { établissement de soins de santé. }\end{array}$ \\
\hline 18 & C.-B. & 2 & $\begin{array}{c}9 \\
(2)\end{array}$ & B3 - Harare* & $\begin{array}{l}\text { - La source d'exposition du cas index de cette éclosion } \\
\text { était inconnue. } \\
\text { - Le cas secondaire était un contact familial. }\end{array}$ \\
\hline
\end{tabular}

* Séquences du génotype B3 identiques à la variante de séquence MVi/Harare.ZWE/38.09 (numéro d'enregistrement GenBank : JF973033).

$\dagger$ Séquences du génotype D8 identiques à la variante de séquence MVs/Taunton.GBR/27.12 (numéro d'enregistrement GenBank : JX984461). 